\title{
ARN: volviendo a la vida civil ${ }^{1}$
}

Entrevista a María Isabel Barón

Coordinadora Regional del Valle del Cauca

Agencia para la Reincorporación y la Normalización (ARN)

Por:

Ana Carolina Palma

García

Laura Villegas

Buitrago

Estudiante de Antropología y

Sociología

ana.palma@correo.icesi.edu.co

lauraville10@hotmail.com

Entrevista recibida el 21/02/2018 y aprobado el 04/03/2018

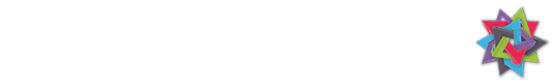

Cómo citar este artículo:

Palma García, A., \& Villegas Buitrago, L. (2018).

ARN: volviendo a la vida civil. Trans-Pasando

Fronteras, (11).

\footnotetext{
${ }^{1}$ El presente documento es el resultado de una entrevista realizada a María Isabel Barón el 30 de Enero de 2018 en la Ciudad de Cali. La Revista Trans-pasando Fronteras le agradece especialmente a ella por su colaboración constante en la construcción de este documento.

2 Profesional en Mercadeo y Negocios Internacionales con Especialización en Gerencia Social. Coordinadora de la ARN desde el 2008. Correo de contacto: mariabaron@acr. gov.co.
} 
Colombia atraviesa un momento de transición clave a raíz del acuerdo de paz con las FARC. Si bien el país se había enfrentado antes a desmovilizaciones colectivas de otros grupos al margen de la ley, esta representa la llegada de masiva de excombatientes del grupo armado más grande del país, que buscan asentarse en las diferentes zonas del territorio nacional. Sin embargo, a diferencia de los prcesos anteriores, en esta ocasión estas personas que buscan reincorporarse y normalizarse en la sociedad civil, cuentan con el acompañamiento de la ARN y su experiencia de 15 años en la implementación de la ruta de reintegración, la cual ha sido reconocida internacionalmente por sus niveles de éxito con un índice de reincidencia de solo el 1\% de quienes han culminado esta ruta exitosamente.

Esta Agencia adelanta procesos de transformación que permitan la superación de la vulnerabilidad y el ejercicio autónomo de la ciudadanía por parte de los excombatientes propiciando políticas participativas, cuya implementación involucra enfoques diferenciales, y atención psicosocial multidimensional, siendo el mayor reto al que se enfrentan la estigmatización por parte del resto de la población civil y el desconocimiento tanto del programa como del esfuerzo que hacen estas personas en busca de una nueva vida en la legalidad.

Esta entrevista toma lugar en Cali, una de las grandes ciudades que con el tiempo se configuró como una de las grandes receptoras de víctimas y excombatientes, en su mayoría del suroccidente del país. Es un lugar clave para pensarse los retos del posconflicto, pues el Valle del Cauca no debe encarar únicamente la llegada masiva de personas desmovilizadas, desplazadas y víctimas, sino que supone también enfrentar los desafíos de una implementación adecuada de las políticas de reincorporación y reintegración a la 
vida civil de estas personas. Cali, pensada en estos términos, es entonces un escenario diverso y complejo cuyas dinámicas, actores, contextos e historias de vida diferentes convergen en un mismo lugar, en un mismo momento histórico. Así pues, el cómo se piensa la intervención ${ }^{3}$ en estos espacios, es lo que motiva este encuentro.

Ahora bien, la presente entrevista se estructura de la siguiente manera, las preguntas fueron seleccionadas por temáticas identificadas en los subtítulos. Después de cada subtítulo, se encuentra las respuestas de la coordinadora Regional del Valle del Cauca de la Agencia para la Reincorporación y la Normalización (ARN), María Isabel Barón. El objetivo de presentar la entrevista en este formato, es mostrar la linealidad del discurso y estimular el interés de las y los lectores.

\section{La Agencia en la historia: cambios y trayectorias.}

Antes de abordar la trayectoria histórica de la Agencia, es pertinente explicar cómo un ex-combatiente llega a nuestra Agencia. Una persona en Colombia se puede desmovilizar de manera individual o de manera colectiva. De manera individual, es cuando una persona que está en un grupo armado y dice "no quiero pertenecer más", deja las armas, se vuela -como decimos- y deserta del grupo. Básicamente esta persona debe presentarse ante el Estado, puede ser ante una iglesia, una defensoría del pueblo o a cualquier instancia gubernamental en la que puede acogerse a

${ }^{3}$ Intervención es entendida aquí según Fantova en "Repensando la intervención social", como aquella actividad formal u organizada que intenta responder a necesidades sociales, incidiendo significativamente en la interacción de las personas, y aspirando a una legitimación pública o social (Fantova, 2007: 186). 
los beneficios del programa de desmovilización como tal, siempre y cuando no haya cometido delitos de lesa humanidad. La desmovilización colectiva, por otra parte, es la que se acuerda con el jefe o comandante de un grupo a través de un acuerdo con el gobierno, como se dio en su momento con las AUC que se hizo a través del Alto Comisionado para la Paz. Las desmovilizaciones colectivas normalmente son acogidas -como está establecido en la norma- por el alto comisionado para la paz; las desmovilizaciones individuales están atendidas a través de un programa de atención humanitaria al desmovilizado (GAHD), manejado por el Ministerio de Defensa. Los anteriores procesos son un filtro por el cual una persona desmovilizada debe pasar para poder que nosotros, como Agencia, la acojamos.

Ahora bien, la Agencia Colombiana para la Reintegración existe hace ya casi 15 años, y en ese transcurso nos hemos ido transformado. Hoy en día contamos con la política de 'reintegración', sin embargo, el primer programa que el gobierno implementó para recibir excombatientes de grupos al margen de la ley se basó en la 'reinserción'. Ese programa, que se consolidó en el 91 con las desmovilizaciones de Quintín Lame, se empezó a desarrollar un programa para estos ex combatientes. En el 2003 se creó el PRVC, el programa para la reincorporación a la vida civil, a cargo del Ministerio del Interior. Este fue el primer programa con este fin creado por el fenómeno de las desmovilizaciones colectivas de las AUC. Fueron principalmente estas desmovilizaciones las que fortalecieron el programa como tal, entre el 2003 y 2005, en el cual se crearon unos beneficios para quienes quisieran desmovilizarse.

EI PRVC (programa para la reincorporación a la vida civil) tenía unos servicios más tercerizados en comparación con los que ten- 
emos hoy en día; no era un programa que los atendía netamente, pues en ese momento las personas solamente firmaban una cuenta de cobro por ser desmovilizados y se les pagaba con esa justificación por dos años. Lo que llamamos CODA es el Comité Operativo para la Dejación de las Armas, que certifica si una persona realmente ha pertenecido a un grupo armado o no. Es muy importante resaltar esto porque en el imaginario de la gente, se piensa que la Agencia es quien decide quién es un desmovilizado o no, cuando en realidad nosotros recibimos del Ministerio de Defensa o Del Alto Comisionado para la paz a quienes hayan pasado por su filtro. Eso nos garantiza a los colombianos que quienes hayan pasado por ahí, realmente estuvieron en el grupo armado.

Este proceso tomó lugar entre 2003 y 2006. En este último año se creó la Alta Consejería para la Reintegración (ACR), a partir de la cual se empezó a construir una política. Como esta política era de gobierno y el objetivo era que se lograra instaurar como una política de Estado, se creó el CONPES 3554 (Política Nacional de reintegración social y económica para personas y grupos armados ilegales) en el cual se da un lineamiento y una base sólida a dicha política para que tuviera una continuidad sin depender del presidente de turno.

Al constituirnos como Alta Consejería, se nos dió de alguna manera una autonomía para generar procesos que buscaran responder a las necesidades de la población. A partir de ahí, esa tercerización de la que hablamos al principio en los procesos, empezó a desaparecer. Pues se contrató un equipo humano que se encargaba de atender a dicha población y se creó lo que llamamos la atención psicosocial. En su mayoría, el equipo fue conformado por psicólogos, porque durante todo este periodo recorrido nos dimos cuenta que la mayoría de personas que estuvieron en un 
espacio o unas dinámicas de guerra, vienen con afectaciones psicológicas que requieren atención. Por ejemplo, hicimos una base de datos cuando los excombatientes entraban al proceso, y nos dimos cuenta de que el $80 \%$ padecen de estrés postraumático o alguna afectación psicológica, debido a su participación en el conflicto armado. Esto fue un reto significativo para la Agencia, porque implicó pensar cómo abordar esta problemática para que logren superar esta condición y reintegrarse a la comunidad como un ciudadano del común. Cuando empezó el proceso, pensamos "si no logramos que esa persona se estabilice, difícilmente vamos a lograr que esa persona vuelva con su familia, vuelva a estudiar, o que sea percibida como una persona con todas las condiciones para reintegrarse".

En el 2011 pasamos a llamarnos Agencia Colombiana para la Reintegración (ACR), porque básicamente, esto nos daba independencia en el tema administrativo y financiero. El ser Agencia nos dejó ser más autónomos en presupuesto, y por tanto en la creación de programas. Esto nos permitía reforzar el proceso que habíamos adelantado hasta el momento, lo cual incrementó los resultados positivos. Debo anotar aquí, que hay algo que nos preocupa mucho a los colombianos y es el índice de reincidencia de los desmovilizados, pues a las personas del común les preocupa que estas personas reintegradas vengan a delinquir a las ciudades. Ese es otro reto que como Agencia hemos tenido que afrontar, el cómo hacerle sentir a los colombianos que una persona que viene de la ilegalidad NO nos va a secuestrar, no va a robar, no va a ser un peligro para el resto.

Finalmente, desde mayo del 2017 el nombre pasó a ser Agencia para la Reincorporación y la normalización (ARN), esto fue complicado porque ya nos reconocían como 'la ACR', así que ahora se 
suma un reto mayor en cuanto a visibilización. El cambio del nombre fue claramente un producto de los acuerdos de paz con las FARC. Los términos 'reincorporación' y 'reintegración' para muchas personas puede significar lo mismo, sin embargo, en la mesa de la Habana se llegó a al acuerdo de optar por 'reincorporación'. Hay algo importante para conocer en esto, y es que las FARC no se desmovilizan, sino que hacen una movilización política, y aquí es donde también tenemos otro reto enfocado al aprendizaje del lenguaje empleado. Es importante resaltar que los cambios con los acuerdos llegan también hasta la parte del lenguaje, porque esto implica que ellos no son desmovilizados como los que venimos atendiendo en la Agencia, de hecho para ellos, quienes veníamos atendiendo son los desertores del grupo, sus enemigos, es decir, el que se salió y trajo información al ejército sobre su grupo.

En eso también es importante aclarar que nosotros no tenemos nada que ver en esas estrategias militares, nosotros somos la parte humana de este proceso, es decir, cuando el desmovilizado llega y me dice "yo sueño con estudiar" o "yo sueño con volver con mi familia", por eso la atención psicosocial es tan importante y fundamental en el proceso.

Estos cambios nos han ayudado al desarrollo de un modelo de atención multidimensional, que busca básicamente que se ayude a estas personas a superar sus condiciones de vulnerabilidad. Todos estos cambios nos han fortalecido para adaptar un modelo, porque la población de nosotros tiene unas particularidades, lo que implica un proceso complejo para lograr construir un modelo lo suficientemente atractivo para que las personas permanezcan y avancen en él, al tiempo que fortalezca las competencias que necesitamos en el tema de la legalidad. 


\section{¿Cómo se posiciona la atención psicosocial en este proceso?}

La atención psicosocial se ve desde un ámbito más amplio, pues nos permite entender al individuo de una manera más integral en su relación con su contexto. Con este modelo buscamos entender los aspectos que le generan vulnerabilidad a este sujeto que estuvo en la guerra y hoy está en el proceso de reincorporación a la vida civil, para poder así ayudarlos a transitar esta ruta, que llamamos ruta de reintegración.

La perspectiva Psicosocial de la ARN se fundamenta en una visión sistémica de la realidad, desde los supuestos de la Teoría Ecológica, no se trata de un abordaje restringido desde un enfoque psicológico particular que excluye otros enfoques en los que pueden estar formados los colaboradores de la ARN, sino más bien una comprensión de los fenómenos psicológicos y sociales a través de los sistemas interdependientes en que se desenvuelven las personas y la comprensión de la realidad como una construcción colectiva en los intercambios relacionales, el objetivo es lograr que las personas en proceso de reintegración se fortalezcan para que logren ser un ciudadanos del común con habilidades y competencias que les permita reintegrarse realmente.

Como mencioné anteriormente, el 80\% padecen de estrés postraumático o alguna afectación psicológica, debido a su participación en el conflicto armado, identificándose problemas de salud mental críticos que requieren de una atención especializada -no son la mayoría-, los cuales serán atendidos por profesionales reintegradores clínicos, quienes a su vez remitirán los casos a las entidades de salud encargadas de la atención de acuerdo con la naturaleza de las afectaciones. 
Para posibilitar el desarrollo de este acompañamiento psicosocial en toda la ruta de reintegración, la ARN cuenta con la Estrategia Multimodal de Incidencia en Salud Mental (EMIS) como una apuesta para transversalizar la perspectiva de salud mental en el proceso de Reintegración, la cual ha sido desarrollada como producto de una alianza entre la Agencia y 3 Universidades de Bogotá.

Esta estrategia, más que tener una cita con un psicólogo o psiquiatra -porque hay casos que lo requieren-, busca entender la salud mental más allá de lo patológico enfatizando en el bienestar del individuo, desde una noción positiva de la salud mental que la aborda como un estado dinámico de bienestar emocional, psicológico y social, obligándonos a pensar cómo hacemos para que sus relaciones con los contextos sean de bienestar y permitan que esta persona pueda desenvolverse bien. Por ejemplo, muchas personas vienen de vivir en un contexto violento y llegan a hacer su reintegración en las zonas más violentas de las ciudades. Es muy compleja esta realidad porque representa un reto adicional el tener que afrontar que alguien que quiera volver a la vida civil, pase de ganarse la vida desde la ilegalidad, a vivir en un barrio con un tema de ilegalidad latente. Tenemos que preguntarnos cómo hacemos para fortalecer su familia, su núcleo, sus redes de apoyo, para que esta persona sienta que tiene un apoyo y pueda estudiar, pueda formarse, y en esa medida pueda ir mejorando sus condiciones.

Hay algo interesante ahí, y es que la atención psicosocial también concibe el trabajo con la familia; nosotros comprobamos que la familia es un factor protector de arraigo a la legalidad, en la medida que la persona tenga buenas redes de apoyo, difícilmente va a ver en el conflicto o una acción violenta una forma de ganarse la 
vida. Muchos de ellos dicen "yo no quiero que mis hijos vuelvan a vivir esto", "yo volví porque quería ver a mi familia, reconstruir los lazos", y ahí también nos encontramos con una serie de retos, y es que muchos de ellos estuvieron 10-15 años en la guerra, entonces cuando vuelven y ya no tienen familia, la esposa no los está esperando, ya se casó, tiene otros hijos... y eso es un choque muy grande para la persona, porque es desertar de un grupo, que es arriesgar su vida completamente, esperando a llegar a encontrar su familia, y cuando llega no está. Por eso la Agencia trabaja fuertemente en el tema de lo psicosocial, de acompañarlos en ese espacio, y ellos lo valoran mucho.

\section{¿Cómo hace la Agencia para que las personas se mantengan en el proceso?}

El éxito es precisamente la atención psicosocial, que no está únicamente desde los psicólogos (que es una parte fundamental), sino que encontramos que la interdisciplinariedad del equipo ayuda al proceso, esta es la parte transversal de la ruta. Lo más importante es que cuando un ex-combatiente llega para el proceso, se le asigna una persona que llamamos reintegrador, que se vuelve como ese referente que lo orienta. El acompañamiento se hace necesario porque el $75 \%$ de las personas vienen de la zona rural a reintegrarse a zonas urbanas, porque acá pueden pasar desapercibidos, en su imaginario está la ciudad como un espacio con más oportunidades. Entonces llegan aquí y no saben ni siquiera pasar un semáforo; para mucha gente puede ser muy tonto, pero ese acompañamiento que hace el reintegrador es fundamental para que esta persona entienda esas lógicas de civilidad, de ciudadanía que son necesarias hasta para coger un bus. 
Lo que planteamos en este proceso o ruta de reintegración, es una atención multidimensional, que contiene ocho dimensiones con base en lo que la experiencia nos ha indicado que son los mayores factores de vulnerabilidad de estas personas. Estas dimensiones son la personal, la familiar, la educativa, la productiva, la de salud, la ciudadana, la de hábitat y la de seguridad. Básicamente esas dimensiones que trabajamos en la ruta, tiene un promedio de duración de 6 años y medio. Un plus de la atención personalizada, es que tenemos un principio y un final. Usualmente se han efectuado procesos asistencialistas que ni buscan una transformación o una movilidad, pero acá, quien inicia el proceso tiene necesariamente un final con el cual el ciclo culmina, como una graduación. Este no es un proceso asistencialista, y los colombianos deben saber eso, porque este es un proceso que se hace con sus recursos, y tenemos derecho a saber si con estos recursos se están generando realmente transformaciones sociales.

\section{Reincidencia: los temores de la desmovilización.}

El nivel de reincidencia, gracias a un estudio de la Fundación Ideas para la $\mathrm{Paz}$, el $76 \%$ de las personas que ingresan a nuestro proceso permanecen en la legalidad, lo que es inversamente proporcional al sistema penitenciario. Esto quiere decir que en el sistema penitenciario, de cada 10 personas que salen, 8 reinciden. Nuestro director hablaba hace poco de lo injusto socialmente que es hacer esa comparación en el sentido que se supone que una persona que estuvo en la cárcel, culminó y salió bien, "civilizado" de nuevo, en este sentido la comparación debe ir con las personas que ya culminaron el proceso de reintegración. También hay que tener en cuenta que es mucho más rentable el proceso de reintegración tanto a nivel social y como a nivel económico. De los 
culminados del proceso de reintegración en el país, solo el 1\% ha reincidido, una tasa mínima en comparación con el sistema penitenciario que es el 80\%. Además, a nivel económico un preso a Colombia le vale al año aproximadamente $\$ 18$ '000.000, mientras un desmovilizado en la ruta, más o menos le vale \$7'800.000. Esta comparación es para que entendamos que el acompañamiento psicosocial de verdad permite que haya ese avance y esos cambios en las personas.

\section{Éxito a nivel internacional}

El proceso de nosotros ha tenido buenos resultados, y son comprobables, de hecho hace muchos años venimos haciendo unas giras internacionales, donde invitamos a expertos de distintos países que han tenido conflicto interno, para intercambiar experiencias. Ya hemos tenido 6 giras, y las conclusiones a las que se se ha llegado, es que Colombia tiene el mejor proceso de reintegración del mundo, y sin embargo hay mucha gente aquí en nuestro país que lo critica o no lo conoce, pero cuando comparamos con países como República Democrática del Congo, Filipinas, Corea, entre otros, y se revisan nuestra política de atención, es claro que es mucho más humana, mejor a largo plazo y de verdad se fija en el individuo, en el humano, la familia y las comunidades que lo reciben.

Al principio trabajamos solo con los ex-combatientes que iniciaban la ruta, los formábamos, se educaban, sin embargo en ese proceso de reintegración la persona llegaba y me decía "mire, yo ya soy bachiller, me gradué de técnico en el SENA, pero resulta que a mí en las empresas no me dan empleo porque soy desmovilizado" o "en mi barrio se dieron cuenta de que yo soy desmovilizado y me 
están echando, porque todo lo que se pierde en el barrio, creen que fui yo, por desmovilizado". Ahí fue que nosotros pensamos que no nos ganábamos nada con formarlos, con lograr una transformación, si los entornos donde están conviviendo son entornos expulsores. Muchos dicen "me va a tocar delinquir", que no debe ser nunca la opción, pero dicen "mire, mis hijos están aguantando hambre, yo me fui a la guerra porque aguantaba hambre, hoy me formo y me siguen dando la espalda". Ahí nosotros debemos entrar a trabajar también en sensibilizar a los empresarios, a las comunidades, etc., para mostrarles con casos reales que personas que estuvieron 20-25 años en estos grupos armados, hoy pueden decir que tomaron una decisión equivocada en su momento y que de verdad quieren construir un proyecto de vida en la legalidad. Al trabajar con desmovilizados este precisamente es el verdadero reto de la reintegración,

Hace dos años tuvimos que hacer una jornada de sensibilización con los familiares de los colaboradores de la Agencia, porque muchos llevaban bastante tiempo trabajando cuando incluso sus familias no comparten esto, sienten miedo, piensan que es peligroso, pero realmente yo nunca me he sentido en riesgo. Lo más difícil no es trabajar con los desmovilizados, lo más difícil es que la gente en Colombia entienda que tenemos un conflicto con unas causas estructurales, que va más allá de buenos y malos, el problema es la estigmatización. Nosotros estamos haciendo un esfuerzo por demostrarles con los 60.000 desmovilizados, que realmente son personas que hoy como cualquiera, estudian, trabajan, tienen familia. Lo más difícil es que la gente entienda que hay que darle una segunda oportunidad a quienes de verdad están comprometidos a construir un proyecto de vida en la legalidad. 


\section{¿Cómo se maneja el proceso con las persona que inician pero no se adhieren del todo al proceso?}

También les quiero contar que como estrategia, quienes no cumplen, tienen unas sanciones o pérdida de beneficios como lo llamamos nosotros. Porque digamos que no puede generarse un mensaje a la comunidad de "yo tengo que ser desmovilizado para que el Estado me cumpla o me de". Esta es una política condicionada al esfuerzo, y por eso tiene un seguimiento. A algunos se les dan unos incentivos por participar en la ruta, que le permiten estabilizarse mientras adquiere esas competencias básicas, porque si vienes de un grupo armado, con una mano adelante y otra atrás y te digo que tienes que buscar trabajo solo ¿dónde buscas? ¿quien te va a dar un trabajo si no tienes experiencia, hoja de vida, si apenas vas a sacar la cédula? El proceso tiene que contar con unos incentivos, no tan mínimos como para que no generen motivación, pero tampoco tan altos, que generen que la persona no quiera esforzarse a avanzar. En promedio en la ruta, una persona puede recibir $\$ 320.000$. Estos beneficios tienen un tiempo, por ejemplo, una persona que entra analfabeta, el proceso le da un lapso de 6.5 años con apoyo económico para que logre aprobar primaria y secundaria bajo un modelo de educación flexible para adultos. Si no le va bien, el apoyo no va a ser para siempre, se permite que una persona sin ningunas discapacidades cognitivas pierda un ciclo (ciclos lectivos especiales integrados CLEI), pero si vuelve a perder el mismo ciclo pierde el apoyo. Eso es importante porque el $67 \%$ de las personas en el proceso ya no recibe ningún apoyo económico y aún así asisten a todas las actividades. Nosotros los vemos al menos una vez al mes, y asisten sin que el Estado le pague por eso, asisten porque reconocen que hay una estrategia psicosocial que les genera beneficios para ellos y sus familias. Es muy valioso porque la gente piensa que van solo cuando 
se les paga, pero lo que hemos ganado en este acompañamiento con el equipo, es que las personas vean un beneficio más allá de lo económico.

Hay muchos que te pueden decir que fueron altos mandos y manejaban mucho dinero en el grupo, y hoy ganan el mínimo, pero te dicen, "yo nunca me devolvería, así me paguen mil millones, yo estoy tranquilo aquí con mi familia, no quiero volver allá". Recuerden que en la actual ruta del proceso de paz hay unos beneficios que corresponden al 90\% de un salario mínimo por 24 meses, pero sólo si no encuentra trabajo antes. Sin embargo, lo que buscamos por esos 24 meses no es que se queden sin hacer nada, que ellos aprovechen ese tiempo para formarse, para volver con su familia, etc.

\section{¿Cómo se manejan las diferentes prácticas o conocimientos que traen estas personas al proceso?}

Algo muy interesante de la estrategia psicosocial es que no desconocemos que hay una historia. El haber pertenecido a un grupo armado no quiere decir que todo lo que aprendieron está mal, son personas que de hecho aprendieron unas competencias que si bien se usaron en la ilegalidad, nosotros las fortalecemos en contextos de legalidad. El que dice "yo era el enfermero del grupo" y me dice que quiere ser médico y tiene todas las competencias, decimos listo, apoyemoslo; o el que era líder porque tenía una formación política, valorada en grupos como las FARC y de guerrilla, tienen una formación bien interesante y muchos quieren participar en espacios políticos, entonces como apenas están escalando estos ámbitos, participan en la junta de acción comunal, o son personas que se reconocen como líderes comunitarios. Precisa- 
mente en eso trabajamos en la dimensión ciudadana, en potenciar todas esas competencias que tienen para que se formen en estas.

Entonces no se desconocen estos aspectos durante el proceso, esos activos con los que ellos llegan desde su contexto y buscamos potenciarlos. Alguna vez alguien le dijo a un desmovilizado "estudie para que sea alguien en la vida" y este le respondió "¿usted cree que yo no soy nadie en la vida? yo era el mejor explosivista de mi frente, yo sí soy alguien en la vida", ese tipo de situaciones hacen que uno piense "pilas, porque es que él no viene de cero". Aquí se hace importante aprender a ver esa línea base de los conocimientos que tiene esta persona para ayudarlos a canalizar esas habilidades. Por ejemplo, si fue el mejor explosivista, entonces tiene habilidades con la motricidad fina y demás, así que podemos mirar cómo puede usar eso para trabajar en mecánica o un oficio así. Es, como decía antes, redireccionar los liderazgos y las capacidades de estas personas hacia la legalidad.

\section{¿Cómo es la culminación del proceso? ¿cuándo se puede decir que alguien está reintegrado a la sociedad?}

Otro tema también importante es el acompañamiento Post que se les hace, así ya no tengan ningún vínculo con la Agencia. El proceso tiene un inicio y un final. Cuando un desmovilizado llega, transita la ruta y se forma, ¿cómo se culmina su proceso? ¿cuál es el reintegrado ideal? ¿cuál es el índice de reintegración? Nosotros nos dimos cuenta que esto es diferente e inherente a cada persona. Tenemos casos de personas que llegan hasta 'Ciclo 2' (ciclos lectivos especiales integrados CLEI) donde aprenden a leer y a escribir, y no quieren estudiar más porque quieren dedicarse a otras actividades como las del campo. Si nosotros vemos que es 
una persona sostenible, que tiene su familia, su red de apoyo, se gana la vida con su actividad agrícola, ya hizo servicio social, y la comunidad ya lo reconoce, consideramos que es una persona ya reintegrada con éxito.

Este proceso supuso entender también su realidad, sus activos y habilidades, para que sean ellos mismos los que decidan hasta dónde quieren llegar. Por eso el modelo propende por el ejercicio autónomo de la ciudadanía. Una vez esta persona se culmina -es decir que ya no es una carga para el Estado, que es un promotor de desarrollo en su comunidad, que es un colombiano más- nosotros tenemos una estrategia que consiste en que por dos años mas, les hacemos un acompañamiento para medir el impacto que el proceso de la ruta y el acompañamiento psicosocial han tenido en sus vidas. Algunas veces nos han preguntado por qué hemos culminado a personas que se han quedado sin trabajo, pero eso pasa por la cuestión que en la vida real eso también le puede pasar incluso al mejor profesional. Lo que quiere decir esto es que este colombiano reintegrado por más que se quede desempleado, va a poder sortear la situación porque ya tiene las habilidades y competencias necesarias para defenderse.

Lo que hemos logrado ha tenido resultados muy positivos. De 20.000 culminados, únicamente 34 han reincidido, lo que representa menos del $1 \%$. Además, hay una probabilidad del 23.5\% de morir por homicidio para aquellas personas que no ingresaron al proceso y solo del $7 \%$ de quienes sí lo hicieron. Porque se debe tener en cuenta que de esas 60.000 personas que tenemos hoy en desmovilización no todas entraron al proceso que supone la ruta, porque el inicio es un proceso voluntario.

Aquí les presentamos una serie de garantías que les permite a ellos hacer su ruta, lo que más genera preocupación es el tema de 
seguridad. Si bien nosotros no somos la entidad que presta la seguridad para ellos, si somos el ente llamado a coordinar con las entidades correspondientes. Para el tema psicológico es igual, si una persona tiene un situación de salud mental crítica, diagnosticado y que requiere de medicación -como la esquizofrenia-, nosotros no lo hacemos porque no manejamos terapia clínica, nosotros estamos llamados a trabajar de manera corresponsable con lo entes a los que sí les corresponde (por ejemplo el hospital psiquiátrico a través de la red pública de salud para ver cómo se les atienden esas necesidades de salud mental).

\section{¿Qué cambios ha tenido la institución o que cambios de- bería tener frente a estos retos que surgen a raíz del acuerdo de paz?}

Yo te hablo desde la Agencia. Se han creado varias instituciones por el posconflicto, parte de eso responde a la implementación del acuerdo. Frente a esto, uno de los cambios de la Agencia ha sido el cambio de nombre (ACR a ARN) y del lenguaje empleado. Si bien venimos haciendo una política regular de atención a desmovilizados, hoy estamos construyendo una política junto a las FARC de manera concertada para idear cuál es la mejor política para atenderlos a ellos y a sus familias de acuerdo a las expectativas que están dentro del acuerdo. Internamente también hemos tenido unos cambios. Tenemos un equipo que se llama "equipo de proyectos especiales" que está pensando hoy junto con las FARC ese modelo de atención.

Hoy nos vemos retados a pensar un modelo que responda a temas desde lo colectivo y no desde lo individual como lo veníamos haciendo. Ya no se trata de ayudarle a alguien a hacer una 
tienda, sino que ahora el antes integrante FARC que hace parte del partido político plantea el desarrollo de un proyecto en común (de gallinas y peces, por ejemplo) donde se beneficien a 20 personas que viven en un mismo lugar con un ideal colectivo. Esa transformación la tenemos que hacer. Y no sólamente ha sido un reto para nosotros como Agencia, sino para todas las instituciones estatales. Por ejemplo, la Agencia nacional de Tierras se había pensado la restituciones de tierras desde lo individual, pero ahora tiene que pensar ¿cómo lo hacemos desde lo colectivo y en sintonía con la reforma agraria integral?; otro ejemplo son las administraciones locales, pues se pensaban que las comunidades debían ir hasta las oficinas en las grandes ciudades mientras que ahora se han tenido que ir donde están en este momento: los Espacios Territoriales de Capacitación y Reincorporación, que en su momento se llamaron Zonas Veredales. Entonces, hay una serie de cambios a nivel estructural que también deben asumir las instituciones.

Lo otro consiste en formar a los funcionarios públicos, tienen que dejar las posiciones personales a un lado, pues la función principal es hacer valer la constitución que defiende la igualdad y la no discriminación de la diferencia. Porque desafortunadamente existen muchos -incluso en el Ministerio público que es el defensor de los derechos- que se niegan a atender a desmovilizados porque atienden víctimas, cuando estas aseveraciones no son posibles porque a los desmovilizados al ser sujetos de derechos y deberes dentro de la constitución, se les debe igualmente proteger y garantizar sus derechos. Es fundamental formar a las personas para ejercer sus cargos desde estas funciones y no desde posturas polarizadas.

Nosotros como Agencia nos hemos visto obligados a afrontar el reto de hacer pedagogía al interior de las instituciones públicas 
para que entiendan cuál es su rol como funcionario público en este escenario de posconflicto, o post-acuerdo (muchas persona prefieren llamar a este periodo de esta forma porque consideran que el conflicto es algo inherente al ser humano y que en parte es necesario para movilizar los cambios y las transformaciones). Otro cambio significativo ha sido el del lenguaje empleado en la Agencia, y en general a nivel las demás instituciones. Por ejemplo, el denominar este periodo de transición como post-acuerdo y no posconflicto.

Este año se llevó a cabo el Censo Nacional del DANE. Las discusiones que se han dado alrededor de este han girado en torno a las poblaciones que al no ser incluidas podrían invisibilizarse más adelante en materia de políticas públicas e intervenciones (esto en lógica de "lo que no se cuenta no existe"). Ejemplo de esto, son las poblaciones campesinas, y LGTB+. ¿Cómo se ubican ahí los desmovilizados? ¿se han dado discusiones alrededor de este tema?

Hasta ahora no han dicho que figuren dentro de las exclusiones del Censo, pero hay algo bien interesante detrás de este tema, porque resulta que uno puede ser desmovilizado pero también ser discapacitado, entonces ¿cómo se clasificaría a esa persona? En situaciones como esto hay unos sesgo de la información, porque por ejemplo, hay personas que tiene varias condiciones diferenciales que hay que tener en cuenta. Para nosotros, un tema clave en la atención psicosocial ha sido fundamental tener en cuenta los enfoques diferenciales, trabajamos entonces temas como género, etnias, desvinculados (Aquel menor de 18 años que ha participado en las acciones de guerra orientadas por un grupo armado irregular y ha sido capturado, se ha entregado voluntariamente o ha sido entregado por el grupo armado irregular siendo menor de edad), discapacidad, afectaciones de salud particulares 
(como enfermedades de alto costo) y consumo. Por ejemplo, en el Valle del cauca no tenemos una población significativamente alta de indígenas, pero sí lo tenemos de poblaciones afrodescendiente, y eso implica que nuestro modelo aquí debe tener claro a qué responde esa población para poder adaptar el modelo de forma eficiente. También nos encontramos con quienes entran con doble condición, de desmovilizado y víctimas, esto es muy importante tenerlo en cuenta en los enfoques de la Agencia.

Algunos de los que están actualmente en la ruta nos han preguntado cómo reportarse en este Censo, ¿como desmovilizado? yo creo que eso lo decide cada uno. Pero la verdad es un tema que actualmente todavía no está claro, supongo que si se incluye a las víctimas también deben incluirse a los desmovilizados. Ahí debieron poner un item aparte donde dijera por ejemplo a qué otro tipo de grupo poblacional pertenece, para ver también esa interseccionalidad. Lo que dicen es que el excluirlos evita que no sean incluidos en la generación de políticas públicas, que es lo que generalmente es el objetivo del Censo, pero de hecho nosotros le pasamos toda la información a la presidencia, como de ubicación, edad, y demás datos de la línea base, para que -me imagino- ellos lo crucen. Pero de todas maneras pasa que muchos de ellos, se han reportado como víctimas, porque por ejemplo, muchos de ellos fueron desplazados, o el día que los reclutaron desplazaron a su familia, y como reportan por núcleo familiar, ellos también están como víctimas. Esto hace que a veces haya una pelea en los espacios institucionales donde unos dicen "esto es de víctimas y no de desmovilizados". Además, hay un pensamiento -bastante equivocado- donde algunas victimas dicen "no es que los desmovilizados tienen más beneficios que las víctimas" y la Ley 1448 es supremamente clara en que hay muchos beneficios para las víctimas, lo que pasa es que son muchas. 
Nosotros tenemos aprox. 60.000 desmovilizados hoy mientras ellos son más de 4'000.000 de víctimas, imaginense la desproporción que hay. Una situación relacionada a esto pasó por ejemplo hace unos dos años, con el proyecto de Gestores de Movilidad en el Tránsito, donde el alcalde dijo que 50 puestos eran para desmovilizados y 50 para víctimas, pero eso despertó una discusión alrededor del nivel de proporción para no generar inequidad ni exclusión social a uno de estos grupos.

\section{¿Cómo es el manejo de los menores de edad que se desmo- vilizan? ¿son ellos quienes usualmente entran con esa doble condición?}

Lo menores de edad son atendidos inicialmente por el ICBF, tiene la ruta de restablecimiento de derechos por ser menores de edad. Una vez cumple la mayoría de edad, son entregados a la Ruta, y ellos, como desvilculados (no desmovilizados), son atendidos tanto por la Agencia como la unidad de atención a las víctimas porque ellos son sujetos de reparación administrativa.Así el menor de edad diga que se fue por voluntad propia y no porque lo obligaron, para el Estado, estas personas no están en todas sus facultades de tomar esa decisión y por tanto se declara de todas maneras víctima. Por esta razón, para los menores de edad que se vinculan a la Agencia se trabaja un enfoque diferencial que tenga en cuenta todo este proceso.

\section{¿Cómo se ha adaptado Cali?}

Estoy convencida de que Cali es pionera en términos de procesos inclusivos, por ejemplo, el programa de gestores de paz 
liderado por la Secretaria de Paz y Cultura Ciudadana de la Alcaldía de Cali, y como ganancia es que también nos están Ilamando de distintas administraciones porque quieren que les pasemos nuestro modelo para trabajar con jóvenes vulnerables, es decir, con jóvenes que han estado en pandillas. El modelo se adapta a muchas características de esta población, claramente se le realizan unos cambios, porque un pandillero no tiene las mismas motivaciones que una persona que se fue a un grupo armado, pero las condiciones de vulnerabilidad sí son muy similares. Entonces, hoy estamos haciendo un convenio con la alcaldía para transferirles nuestro modelo, el cual ahora están utilizando con el Programa de gestores de paz, y les ha ido muy bien. De hecho, ha disminuido el índice de violencia, pues ellos han contado, por ejemplo, un indicador que me parece muy chevere. Y es, "bueno y uno como mide para saber si este proyecto está funcionando o no con los jóvenes", entonces ellos tienen un concepto que es: "yo me parcho con el parche", ya que ellos todo el día se parchaban en sus barrios con sus combos. Entonces a estos muchachos -de los cuales muchos pertenecían a pandillas- les empezaban a preguntar "bueno, vos cuántas veces te parchabas con el parche en tu cuadra", y los resultados se evidenciaban en que ellos respondían "no pues, antes, todos los días en la tarde y ahora solo una vez al mes". Entonces eso de alguna manera, disminuye tu vulnerabilidad a esos grupos, porque el pasar de que tú estés todos los días, a que tú ya solo estés una vez, significa que el resto del tiempo lo estás usando de manera productiva.

La idea es lograr que la Agencia algún día desaparezca, esperamos que algún día se acaben los desmovilizados y no haya más conflicto interno, que logremos reintegrar a todas las personas, pero que aún así, no se pierda este modelo. Entonces por esto la idea es lograr compartirlo con otras poblaciones que lo podrían necesi- 
tar, porque creo que nuestro problema, más que los excombatientes es la violencia urbana. Hoy nos decían: "bueno, ya estamos solucionando el problema del conflicto armado ¿ahora a quién le vamos a echar la culpa de la inseguridad? Por lo tanto, ahora las alcaldías tienen más posibilidades de repensarse realmente qué está generando los problemas de violencia en las urbes, y se dieron cuenta de que la violencia no la explican los desmovilizados, tenemos narcotráfico, tenemos una historia de desplazamiento, tenemos una historia de violencia, pero la única razón no son los desmovilizados. 